\title{
Directed Flow of Charged Particles at Midrapidity Relative to the Spectator Plane in Pb-Pb Collisions at $\sqrt{s_{\mathrm{NN}}}=2.76 \mathrm{TeV}$
}

\author{
B. Abelev et al.* \\ (ALICE Collaboration)
}

(Received 18 June 2013; published 6 December 2013)

\begin{abstract}
The directed flow of charged particles at midrapidity is measured in $\mathrm{Pb}-\mathrm{Pb}$ collisions at $\sqrt{s_{\mathrm{NN}}}=$ 2.76 TeV relative to the collision symmetry plane defined by the spectator nucleons. A negative slope of the rapidity-odd directed flow component with approximately 3 times smaller magnitude than found at the highest RHIC energy is observed. This suggests a smaller longitudinal tilt of the initial system and disfavors the strong fireball rotation predicted for the LHC energies. The rapidity-even directed flow component is measured for the first time with spectators and found to be independent of pseudorapidity with a sign change at transverse momenta $p_{T}$ between 1.2 and $1.7 \mathrm{GeV} / c$. Combined with the observation of a vanishing rapidity-even $p_{T}$ shift along the spectator deflection this is strong evidence for dipolelike initial density fluctuations in the overlap zone of the nuclei. Similar trends in the rapidity-even directed flow and the estimate from two-particle correlations at midrapidity, which is larger by about a factor of 40, indicate a weak correlation between fluctuating participant and spectator symmetry planes. These observations open new possibilities for investigation of the initial conditions in heavy-ion collisions with spectator nucleons.
\end{abstract}

DOI: 10.1103/PhysRevLett.111.232302

PACS numbers: 25.75.-q, 25.75.Ld

The goal of the heavy-ion program at the Large Hadron Collider (LHC) is to explore the properties of deconfined quark-gluon matter. Anisotropic transverse flow is sensitive to the early times of the collision, when the deconfined state of quarks and gluons is expected to dominate the collision dynamics (see reviews [1-3] and references therein), with a positive (in-plane) elliptic flow as first observed at the Alternating Gradient Synchrotron (AGS) $[4,5]$. A much stronger flow was subsequently measured at the Super Proton Synchrotron (SPS) [6], Relativistic Heavy Ion Collider (RHIC) [7-9], and recently at the LHC [10-12]. Elliptic flow at RHIC and the LHC is reproduced by hydrodynamic model calculations with a small value of the ratio of shear viscosity to entropy density [13-16]. Despite the success of hydrodynamics in describing the equilibrium phase of matter produced in a relativistic heavy-ion collision, there are still large theoretical uncertainties in determination of the initial conditions. Significant triangular flow measured recently at RHIC $[17,18]$ and LHC $[12,19,20]$ energies has demonstrated $[21,22]$ that initial energy fluctuations play an important role in the development of the final momentum-space anisotropy of the distribution of produced particles.

The collision geometry is illustrated in Fig. 1, which depicts the participant overlap region and spectators as

*Full author list given at the end of the article.

Published by the American Physical Society under the terms of the Creative Commons Attribution 3.0 License. Further distribution of this work must maintain attribution to the author(s) and the published article's title, journal citation, and DOI. viewed in (a) the reaction plane and (b) the plane perpendicular to the beam. Figure 1(a) shows the projectile and target spectators repelled in the reaction $(x z)$ plane from the center of the colliding system along the impact parameter $(x)$ direction. An alternative scenario where spectators
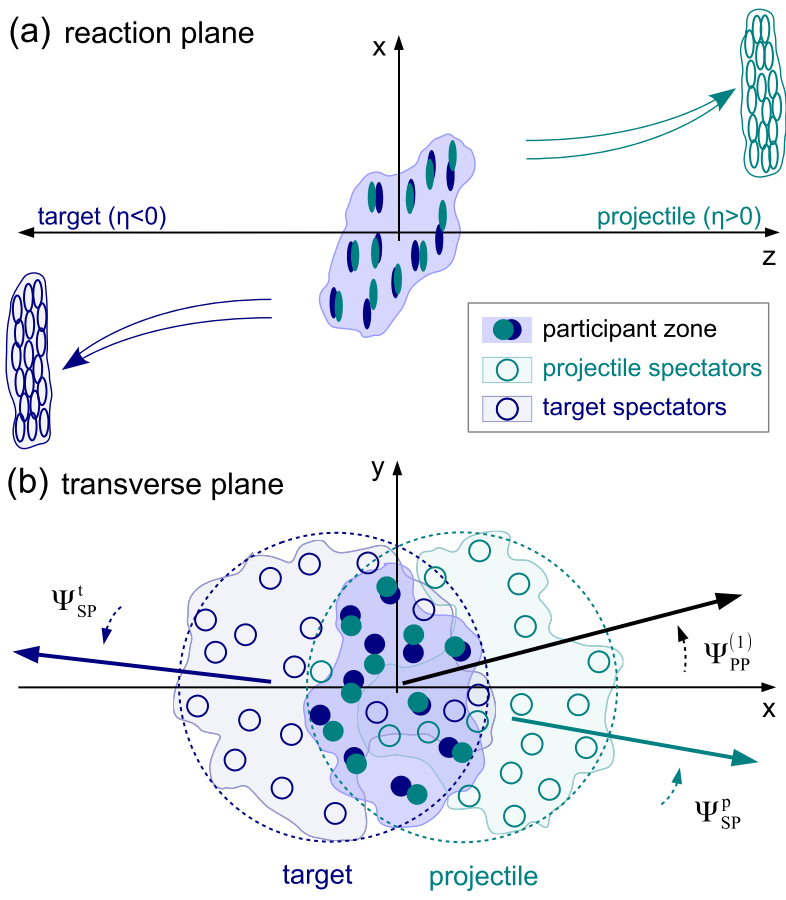

FIG. 1 (color online). Sketch of a noncentral heavy-ion collision. See text for description of the figure. 
are attracted towards the center of the system is discussed in [23].

The directed flow is characterized by the first harmonic coefficient $v_{1}$ in a Fourier decomposition of the particle azimuthal distribution with respect to one of the collision symmetry planes, $\Psi$, as illustrated in Fig. 1(b) and discussed below

$$
v_{1}\left(\eta, p_{T}\right)\{\Psi\}=\langle\cos (\phi-\Psi)\rangle .
$$

Here $\eta=-\ln [\tan (\theta / 2)], p_{T}, \theta$, and $\phi$ are the particle pseudorapidity, transverse momentum, polar, and azimuthal angles, respectively. The brackets " $\langle\ldots\rangle$ " indicate an average over measured particles in all recorded events.

For a nonfluctuating nuclear matter distribution, the directed flow in the participant zone develops along the impact parameter direction. The collision symmetry requires that the directed flow be an antisymmetric function of pseudorapidity, $v_{1}^{\text {odd }}(\eta)=-v_{1}^{\text {odd }}(-\eta)$. Because of event-by-event fluctuations in the initial energy density of the collision, the participant plane angle $\left(\Psi_{\mathrm{PP}}^{(1)}\right)$ defined by the dipole asymmetry of the initial energy density [24,25] and that of projectile $\left(\Psi_{\mathrm{SP}}^{p}\right)$ and target $\left(\Psi_{\mathrm{SP}}^{t}\right)$ spectators are different from the geometrical reaction plane angle $\Psi_{\mathrm{RP}}[x$ axis in Fig. 1(b)]. As a consequence, the directed flow can develop [24-27] a rapidity-symmetric component, $v_{1}^{\text {even }}(\eta)=v_{1}^{\text {even }}(-\eta)$, which does not vanish at midrapidity.

The slope of $v_{1}^{\text {odd }}$ as a function of rapidity at AGS [5,28] and SPS [29,30] energies is driven by the difference between baryon and meson production and shadowing by the nuclear remnants. At higher (RHIC) energies a multiple zero crossing of $v_{1}^{\text {odd }}$ with rapidity outside the nuclear fragmentation regions was predicted as a signature of the deconfined phase transition [31,32]. However, the RHIC measurements [33-36] did not reveal such a structure. The magnitude of the directed flow depends on the amount of baryon stopping in the nuclear overlap zone [37]. The two can be related via realistic model calculations, making $v_{1}^{\text {odd }}$ an important experimental probe of the initial conditions in a heavy-ion collision. The initial conditions assumed in model calculations of $v_{1}^{\text {odd }}$ range from incomplete baryon stopping [37], with a positive space-momentum correlation, to full nucleon stopping with a tilted [32,38] or rotating [39] source of matter produced in the overlap zone of the nuclei. Model calculations generally agree on the negative sign of the $v_{1}^{\text {odd }}$ slope as a function of pseudorapidity at RHIC [33-36]. The model predictions for $v_{1}^{\text {odd }}$ at the LHC vary from having the same slope as at RHIC but with smaller magnitude [38] to an opposite (positive) slope with significantly larger magnitude $[39,40]$.

The $v_{1}^{\text {even }}$ estimated from the two-particle azimuthal correlations at midrapidity for RHIC [41] (see also [25]) and LHC $[12,20,42]$ energies is in approximate agreement with ideal hydrodynamic model calculations $[26,27]$ for dipolelike [24] energy fluctuations in the overlap zone of the nuclei. Interpretation of the two-particle correlations is complicated due to a possibly large bias from correlations unrelated to the initial geometry (nonflow) and due to the model dependence of the correction procedure for effects of momentum conservation [27]. The directed flow measured relative to the spectator deflection is free from such biases and provides a cleaner probe of the initial conditions in a heavy-ion collision. It also allows for a study of the main features of the dipolelike energy fluctuations such as a vanishing transverse momentum shift of the created system along the direction of the spectator deflection. Directed flow and its fluctuations also play an important role in understanding effects due to the strong magnetic field in heavy-ion collisions [24] and interpretation of the observed charge separation relative to the reaction plane [43] in terms of the chiral magnetic effect [44].

In this Letter, we report the charged particle directed flow measured relative to the deflection of spectator neutrons in $\mathrm{Pb}-\mathrm{Pb}$ collisions at $\sqrt{s_{\mathrm{NN}}}=2.76 \mathrm{TeV}$. About $13 \times$ $10^{6}$ minimum-bias [10] collisions in the 5\%-80\% centrality range were analyzed. For the most central $(0 \%-5 \%)$ collisions, the small number of spectators does not allow for a reliable reconstruction of their deflection. Two forward scintillator arrays (VZERO) [45] were used to determine the collision centrality. Charged particles reconstructed in the time projection chamber (TPC) [46] with $p_{T}>0.15 \mathrm{GeV} / c$ and $|\eta|<0.8$ were selected for the analysis.

The deflection of neutron spectators was reconstructed using a pair of zero degree calorimeters (ZDC) [47] with $2 \times 2$ segmentation installed $114 \mathrm{~m}$ from the interaction point on each side, covering the $|\eta|>8.78$ (beam rapidity) region. A typical energy measured by both ZDCs for $30 \%-$ $40 \%$ centrality is about $100 \mathrm{TeV}$ [48]. The spectator deflection in the transverse plane was measured with a pair of two-dimensional vectors

$$
\mathbf{Q}^{t, p} \equiv\left(Q_{x}^{t, p}, Q_{y}^{t, p}\right)=\sum_{i=1}^{4} \mathbf{n}_{i} E_{i}^{t, p} / \sum_{i=1}^{4} E_{i}^{t, p},
$$

where $p(t)$ denotes the ZDC on the $\eta>0(\eta<0)$ side of the interaction point, $E_{i}$ is the measured signal, and $\mathbf{n}_{i}=$ $\left(x_{i}, y_{i}\right)$ are the coordinates of the $i$ th ZDC segment. An asymmetry of $0.1 \%$ [49] in energy calibration of the two ZDCs and an absolute energy scale uncertainty cancel in Eq. (2). To compensate for the run-dependent variation of the LHC beam crossing position, an event-by-event correction $\mathbf{Q}^{t, p} \rightarrow \mathbf{Q}^{t, p}-\left\langle\mathbf{Q}^{t, p}\right\rangle$ [3] was applied as a function of collision centrality and transverse position of the collision vertex relative to the center of the ALICE detector. Experimental values of the correction for the 30\%-40\% centrality class are $\left\langle Q_{x(y)}^{p}\right\rangle \approx 2.0(-1.5) \mathrm{mm}$ and $\left\langle Q_{x(y)}^{t}\right\rangle \approx$ $-1.1(0.01) \mathrm{mm}$.

The directed flow is determined with the scalar product method $[3,50]$ 
$v_{1}\left\{\Psi_{\mathrm{SP}}^{p}\right\}=\frac{1}{\sqrt{2}}\left[\frac{\left\langle u_{x} Q_{x}^{p}\right\rangle}{\sqrt{\left|\left\langle Q_{x}^{t} Q_{x}^{p}\right\rangle\right|}}+\frac{\left\langle u_{y} Q_{y}^{p}\right\rangle}{\sqrt{\left|\left\langle Q_{y}^{t} Q_{y}^{p}\right\rangle\right|}}\right]$,

$v_{1}\left\{\Psi_{\mathrm{SP}}^{t}\right\}=-\frac{1}{\sqrt{2}}\left[\frac{\left\langle u_{x} Q_{x}^{t}\right\rangle}{\sqrt{\left|\left\langle Q_{x}^{t} Q_{x}^{p}\right\rangle\right|}}+\frac{\left\langle u_{y} Q_{y}^{t}\right\rangle}{\sqrt{\left|\left\langle Q_{y}^{t} Q_{y}^{p}\right\rangle\right|}}\right]$,

where $u_{x}=\cos \phi$ and $u_{y}=\sin \phi$ are defined for charged particles at midrapidity. The odd and even components of the directed flow relative to the spectator plane $\left[\Psi=\Psi_{\mathrm{SP}}\right.$ in Eq. (1)] are then calculated from the equations

$$
v_{1}^{\text {odd }}\left\{\Psi_{\mathrm{SP}}\right\}=\left[v_{1}\left\{\Psi_{\mathrm{SP}}^{p}\right\}+v_{1}\left\{\Psi_{\mathrm{SP}}^{t}\right\}\right] / 2
$$

and

$$
v_{1}^{\text {even }}\left\{\Psi_{\mathrm{SP}}\right\}=\left[v_{1}\left\{\Psi_{\mathrm{SP}}^{p}\right\}-v_{1}\left\{\Psi_{\mathrm{SP}}^{t}\right\}\right] / 2 .
$$

Equation (4) defines the sign of $v_{1}^{\text {odd }}$ using the convention used at RHIC $[33,34]$ and implies a positive directed flow [or deflection along the positive $x$-axis direction in Fig. 1(a)] of the projectile spectators.

The negative correlations $\left\langle Q_{x}^{t} Q_{x}^{p}\right\rangle$ and $\left\langle Q_{y}^{t} Q_{y}^{p}\right\rangle$ [51] indicate a deflection of the projectile and target spectators in opposite directions. These correlations are sensitive to a combination of the spectator's directed flow relative to the reaction plane $\Psi_{\mathrm{RP}}$ and an additional contribution due to flow of spectators along the fluctuating $\Psi_{\mathrm{SP}}^{p}$ and $\Psi_{\mathrm{SP}}^{t}$ directions [see Fig. 1(b)]. The two contributions are not separable using current experimental techniques and both should be considered in theoretical interpretations of the results derived from Eqs. (3)-(5). Given that the transverse deflection of spectators $\left[d_{\text {spec }} \approx \sqrt{\left(\left\langle Q_{x}^{t} Q_{x}^{p}\right\rangle+\left\langle Q_{y}^{t} Q_{y}^{p}\right\rangle\right) / 2}\right]$ is tiny compared to the ZDC detector position $\left|z_{\mathrm{ZDC}}\right|=$ $114 \mathrm{~m}$ along the beam direction, one can make a rough estimate of the corresponding transverse momentum carried by an individual spectator: $p_{T}^{\text {spec }} \approx \sqrt{s_{\mathrm{NN}}}\left(d_{\mathrm{spec}} / z_{\mathrm{ZDC}}\right)$. The measured $d_{\mathrm{spec}}$ is about $0.67(0.92) \mathrm{mm}$ [51] for the $5 \%-10 \%(30 \%-40 \%)$ centrality class which yields $p_{T}^{\text {spec }} \sim$ 16(22) $\mathrm{MeV} / c$. Correlations $\left\langle Q_{x}^{t} Q_{y}^{p}\right\rangle$ and $\left\langle Q_{y}^{t} Q_{x}^{p}\right\rangle$ in orthogonal directions, which can be nonzero due to residual detector effects, are less than 5\% [51] of those in the aligned directions. The 10\%-20\% [51] difference between $\left\langle Q_{x}^{t} Q_{x}^{p}\right\rangle$ and $\left\langle Q_{y}^{t} Q_{y}^{p}\right\rangle$ for midcentral collisions is mainly due to a different offset of the beam spot from the center of the ZDCs in plane and perpendicular to the LHC accelerator ring. The corresponding dominant systematic uncertainty is evaluated from the spread of results for different terms in Eq. (3) and estimated to be below 20\%. The results obtained with Eq. (3) are consistent with calculations using the event plane method [3]. The results with opposite polarity of the magnetic field of the ALICE detector are consistent within 5\%. Variation of the results with the collision centrality estimated with the TPC, VZERO, and silicon pixel detectors [47] and with narrowing the nominal $\pm 10 \mathrm{~cm}$ range of the collision vertex along the beam direction from the center of the ALICE detector to $\pm 7 \mathrm{~cm}$ is less than $5 \%$. Altering the selection criteria for the tracks reconstructed with the TPC resulted in a $3 \%-5 \%$ variation of the results. The systematic error evaluated for each of the sources listed above were added in quadrature to obtain the total systematic uncertainty of the measurement.

Figure 2(a) shows the charged particle directed flow as a function of pseudorapidity for $10 \%-20 \%, 30 \%-40 \%$, and $10 \%-60 \%$ centrality classes. The $v_{1}^{\text {even }}(\eta)$ component is found to be negative and independent of $\eta$. The $v_{1}^{\text {odd }}(\eta)$ component exhibits a negative slope as a function of pseudorapidity. This is in contrast to the positive slope expected from the model calculations [39,40] with stronger rotation of the participant zone at the LHC than at RHIC. The $v_{1}^{\text {odd }}(\eta)$ at the highest RHIC energy [34] has the same sign of the slope and a factor of 3 larger magnitude. This is consistent with a smaller tilt of the participant zone in the $x-z$ plane [see Fig. 1(a)] as predicted in [38] for LHC energies. Figure 2(c) compares $v_{1}^{\text {odd }}$ with the STAR data [34] for Au-Au collisions at $\sqrt{s_{\mathrm{NN}}}=200$ (62) GeV downscaled by the ratio $0.37(0.12)$ of the slope at the LHC to

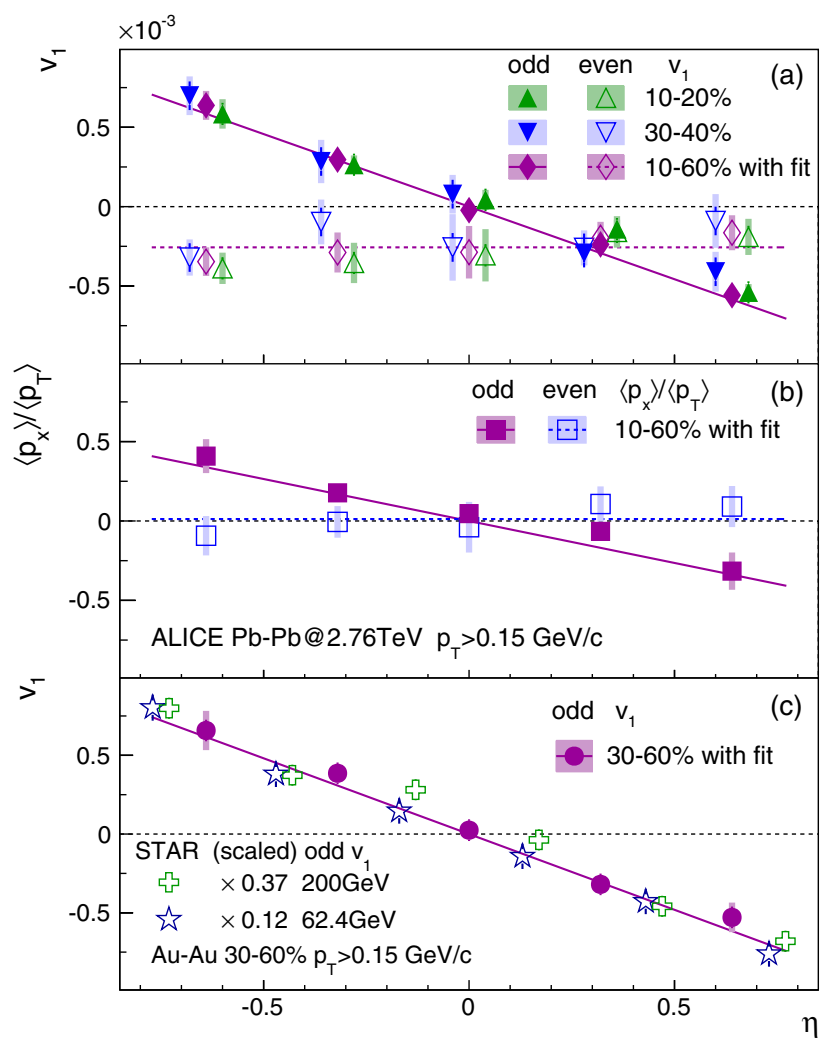

FIG. 2 (color online). (a) $v_{1}$ and (b) $\left\langle p_{x}\right\rangle /\left\langle p_{T}\right\rangle$ versus pseudorapidity in $\mathrm{Pb}-\mathrm{Pb}$ collisions at $\sqrt{s_{\mathrm{NN}}}=2.76 \mathrm{TeV}$. (c) $v_{1}^{\text {odd }}$ compared to the STAR data [34] for Au-Au collisions at $\sqrt{s_{\mathrm{NN}}}=200$ (62.4) $\mathrm{GeV}$ downscaled by a factor $0.37(0.12)$. The statistical (systematic) uncertainties are indicated by the error bars (shaded bands). Lines (to guide the eye) represent fits with a linear (constant) function for $v_{1}^{\text {odd }}\left(v_{1}^{\text {even }}\right)$. 
that at RHIC energy. These ratios indicate a strong violation by a factor of 1.82 (4.55) of the beam rapidity scaling discussed in [36].

Figure 2(b) shows the relative momentum shift $\left\langle p_{x}\right\rangle /\left\langle p_{T}\right\rangle \equiv\left\langle p_{T} \cos \left(\phi-\Psi_{\mathrm{SP}}\right)\right\rangle /\left\langle p_{T}\right\rangle$ along the spectator plane as a function of pseudorapidity. It is obtained by introducing a $p_{T} /\left\langle p_{T}\right\rangle$ weight in front of $u_{x}$ and $u_{y}$ in Eq. (3). The nonzero $\left\langle p_{x}\right\rangle^{\text {odd }} /\left\langle p_{T}\right\rangle$ shift has a smaller magnitude than $v_{1}^{\text {odd }}$. The $\left\langle p_{x}\right\rangle^{\text {even }}$ vanishes which is consistent with the dipolelike event-by-event fluctuations of the initial energy density in a system with zero net transverse momentum. Disappearance of $\left\langle p_{x}\right\rangle$ at $\eta \approx 0$ indicates that particles produced at midrapidity are not involved in balancing the transverse momentum carried away by spectators.

Figures 3(a) and 3(b) present $v_{1}$ and $\left\langle p_{x}\right\rangle /\left\langle p_{T}\right\rangle$ versus collision centrality. The odd components were calculated by taking values at negative $\eta$ with an opposite sign. Both $v_{1}$ components have weak centrality dependence. The $\left\langle p_{x}\right\rangle^{\text {even }}$ component is zero at all centralities, while $\left\langle p_{x}\right\rangle^{\text {odd }} /\left\langle p_{T}\right\rangle$ is a steeper function of centrality than $v_{1}^{\text {odd }}$. This suggests that $v_{1}^{\text {odd }}$ has two contributions. The first contribution has a similar origin as $v_{1}^{\text {even }}$ due to asymmetric dipolelike initial energy distribution. The second contribution grows almost linearly from central to peripheral

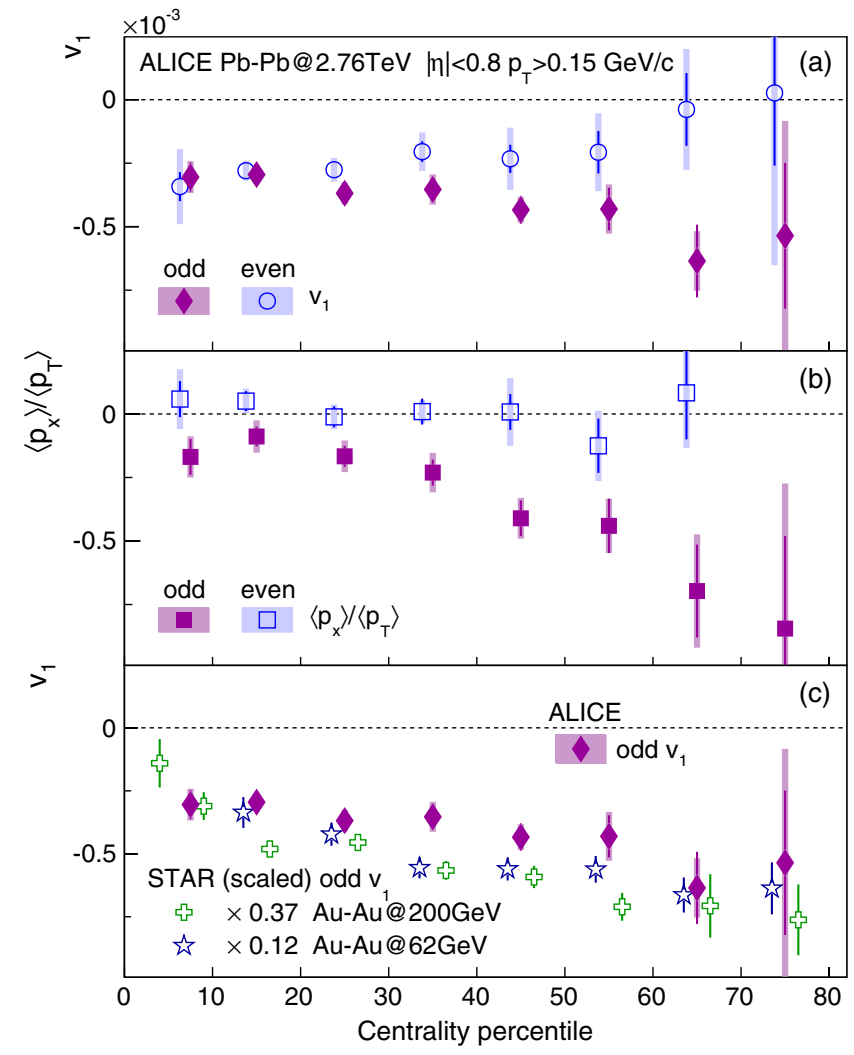

FIG. 3 (color online). (a) $v_{1}$ and (b) $\left\langle p_{x}\right\rangle /\left\langle p_{T}\right\rangle$ versus centrality. (c) $v_{1}^{\text {odd }}$ comparison with STAR data [34]. See text and Fig. 2 for description of the data points. collisions and represents an effect of sideward collective motion of particles at nonzero rapidity due to expansion of the initially tilted source. This $\left\langle p_{x}\right\rangle$ is balanced by that of the particles produced at opposite rapidity and in very forward (spectator) regions. The magnitude of $v_{1}^{\text {odd }}$ at the LHC is significantly smaller than at RHIC with a similar centrality dependence [see Fig. 3(c)].

Figure 4(a) presents $v_{1}$ as a function of $p_{T}$. Both components change sign around $p_{T}$ between 1.2 and $1.7 \mathrm{GeV} / c$ which is expected for the dipolelike energy fluctuations when the momentum of the low $p_{T}$ particles is balanced by those at high $p_{T}$ [24-27]. The $p_{T}$ dependence of $v_{1}^{\text {even }}$ relative to $\Psi_{\mathrm{SP}}$ is similar to that of $v_{1}^{\text {even }}$ relative to $\Psi_{\mathrm{PP}}^{(1)}$ estimated from the Fourier fits of the twoparticle correlations $[12,20,42]$, while its magnitude is smaller by a factor of $40[27,52]$. This can be interpreted as a weak correlation, $\left\langle\cos \left(\Psi_{\mathrm{PP}}^{(1)}-\Psi_{\mathrm{SP}}\right)\right\rangle \ll 1$, between the orientation of the participant and spectator collision symmetry planes. Compared to the RHIC measurements in Fig. 4(b), $v_{1}^{\text {odd }}$ shows a similar trend including the sign change around $p_{T}$ of $1.5 \mathrm{GeV} / c$ in central collisions and a negative value at all $p_{T}$ for peripheral collisions.

According to hydrodynamic model calculations $[24,27,53]$ particles with low $p_{T}$ should flow in the direction opposite to the largest density gradient. This, together with the negative even and odd $v_{1}$ components relative to $\Psi_{\mathrm{SP}}$ measured for particles at midrapidity with low transverse momentum $\left(p_{T} \lesssim 1.2 \mathrm{GeV} / c\right)$ allows one, in principle, to determine if spectators deflect away from or towards the center of the system. However, a detailed

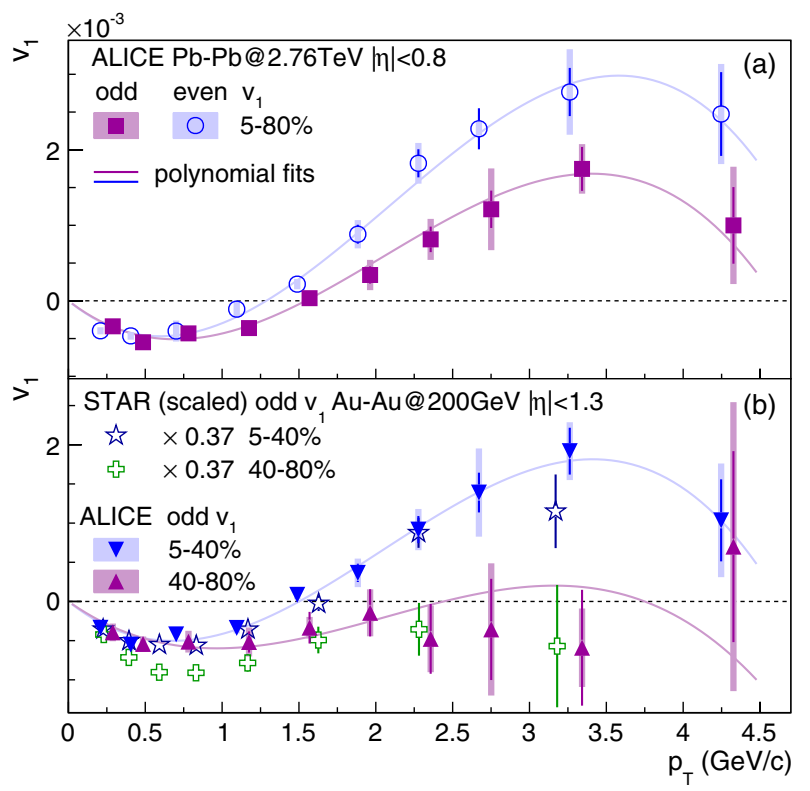

FIG. 4 (color online). (a) $v_{1}$ versus transverse momentum. (b) $v_{1}^{\text {odd }}$ comparison with STAR data [34]. See text and Fig. 2 for description of the data points. Lines (to guide the eye) represent fits with a third order polynomial. 
theoretical calculation of the correlation between fluctuations in the spectator positions and energy density in the participant zone such as in [23] is required to provide a definitive answer to this question.

In summary, the $v_{1}^{\text {odd }}$ and $v_{1}^{\text {even }}$ components of charged particle directed flow at midrapidity, $|\eta|<0.8$, are measured relative to the spectator plane for $\mathrm{Pb}-\mathrm{Pb}$ collisions at $\sqrt{s_{\mathrm{NN}}}=2.76 \mathrm{TeV}$. The $v_{1}^{\text {odd }}$ has a negative slope as a function of pseudorapidity with a magnitude about 3 times smaller than at the highest RHIC energy. This suggests a smaller tilt of the medium created in the participant zone at the LHC, with insufficient rotation to alter the slope of $v_{1}^{\text {odd }}(\eta)$ as predicted in $[39,40]$. As a function of $p_{T}, v_{1}^{\text {odd }}$ and $v_{1}^{\text {even }}$ cross zero at $p_{T}$ between 1.2 and $1.7 \mathrm{GeV} / c$ for semicentral collisions. Disappearance of $\left\langle p_{x}\right\rangle$ for particles produced close to zero rapidity suggest that they do not play a role in balancing the $p_{T}$ kick of spectators. The shape of $v_{1}^{\text {even }}\left(p_{T}\right)$ and a vanishing $\left\langle p_{x}\right\rangle^{\text {even }}$ is consistent with dipolelike fluctuations of the initial energy density in the participant zone. A similar shape but with about 40 times larger magnitude was observed for an estimate of $v_{1}^{\text {even }}\left(p_{T}\right)$ relative to the participant plane from the Fourier fits of the two-particle correlation [12,20,42]. This indicates that fluctuating participant and spectator collision symmetry planes are weakly correlated, which is important experimental input for modeling the ill-constrained initial conditions of a heavy-ion collision. Future studies of the directed flow at midrapidity using identified particles and extension of the $v_{1}$ measurements to forward rapidities should provide a stronger constraint on the effects of initial density fluctuations in the formation of directed flow.

The ALICE Collaboration would like to thank all its engineers and technicians for their invaluable contributions to the construction of the experiment and the CERN accelerator teams for the outstanding performance of the LHC complex. The ALICE Collaboration acknowledges the following funding agencies for their support in building and running the ALICE detector: State Committee of Science, World Federation of Scientists (WFS) and Swiss Fonds Kidagan, Armenia, Conselho Nacional de Desenvolvimento Científico e Tecnológico (CNPq), Financiadora de Estudos e Projetos (FINEP), Fundação de Amparo à Pesquisa do Estado de São Paulo (FAPESP); National Natural Science Foundation of China (NSFC), the Chinese Ministry of Education (CMOE), and the Ministry of Science and Technology of China (MSTC); Ministry of Education and Youth of the Czech Republic; Danish Natural Science Research Council, the Carlsberg Foundation and the Danish National Research Foundation; The European Research Council under the European Community's Seventh Framework Programme; Helsinki Institute of Physics and the Academy of Finland; French CNRS-IN2P3, the "Region Pays de Loire," "Region Alsace," "Region Auvergne," and CEA, France; German BMBF and the
Helmholtz Association; General Secretariat for Research and Technology, Ministry of Development, Greece; Hungarian OTKA and National Office for Research and Technology (NKTH); Department of Atomic Energy and Department of Science and Technology of the Government of India; Istituto Nazionale di Fisica Nucleare (INFN) and Centro Fermi-Museo Storico della Fisica e Centro Studi e Ricerche "Enrico Fermi," Italy; MEXT Grant-in-Aid for Specially Promoted Research, Japan; Joint Institute for Nuclear Research, Dubna; National Research Foundation of Korea (NRF); CONACYT, DGAPA, México, ALFAEC, and the EPLANET Program (European Particle Physics Latin American Network) Stichting voor Fundamenteel Onderzoek der Materie (FOM) and the Nederlandse Organisatie voor Wetenschappelijk Onderzoek (NWO), Netherlands; Research Council of Norway (NFR); Polish Ministry of Science and Higher Education; National Authority for Scientific ResearchNASR (Autoritatea Naţională pentru Cercetare Ştiinţifică-ANCS); Ministry of Education and Science of Russian Federation, Russian Academy of Sciences, Russian Federal Agency of Atomic Energy, Russian Federal Agency for Science and Innovations, and The Russian Foundation for Basic Research; Ministry of Education of Slovakia; Department of Science and Technology, South Africa; CIEMAT, EELA, Ministerio de Economía y Competitividad (MINECO) of Spain, Xunta de Galicia (Consellería de Educación), CEADEN, Cubaenergía, Cuba, and IAEA (International Atomic Energy Agency); Swedish Research Council (VR) and Knut \& Alice Wallenberg Foundation (KAW); Ukraine Ministry of Education and Science; United Kingdom Science and Technology Facilities Council (STFC); USA Department of Energy, USA National Science Foundation, the State of Texas, and the State of Ohio.

[1] W. Reisdorf and H. Ritter, Annu. Rev. Nucl. Part. Sci. 47, 663 (1997)

[2] N. Herrmann, J. Wessels, and T. Wienold, Annu. Rev. Nucl. Part. Sci. 49, 581 (1999).

[3] S. A. Voloshin, A. M. Poskanzer, and R. Snellings, in Landolt-Boernstein, Relativistic Heavy Ion Physics (Springer-Verlag, Berlin, 2010), Vol. 1/23, p. 5.

[4] J. Barrette et al. (E877 Collaboration), Phys. Rev. Lett. 73, 2532 (1994).

[5] J. Barrette et al. (E877 Collaboration), Phys. Rev. C 55, 1420 (1997).

[6] H. Appelshauser et al. (NA49 Collaboration), Phys. Rev. Lett. 80, 4136 (1998).

[7] K. Ackermann et al. (STAR Collaboration), Phys. Rev. Lett. 86, 402 (2001).

[8] K. Adcox et al. (PHENIX Collaboration), Phys. Rev. Lett. 89, 212301 (2002).

[9] B. Back et al. (PHOBOS Collaboration), Phys. Rev. Lett. 94, 122303 (2005). 
[10] K. Aamodt et al. (ALICE Collaboration), Phys. Rev. Lett. 105, 252302 (2010).

[11] S. Chatrchyan et al. (CMS Collaboration), Phys. Rev. C 87, 014902 (2013).

[12] G. Aad et al. (ATLAS Collaboration), Phys. Rev. C 86, 014907 (2012).

[13] P. Huovinen, P. Kolb, U. W. Heinz, P. Ruuskanen, and S. Voloshin, Phys. Lett. B 503, 58 (2001).

[14] U. W. Heinz, C. Shen, and H. Song, AIP Conf. Proc. 1441, 766 (2012)

[15] H. Song and U. W. Heinz, Phys. Lett. B 658, 279 (2008).

[16] H. Song and U. W. Heinz, Phys. Rev. C 77, 064901 (2008).

[17] L. Adamczyk et al. (STAR Collaboration), Phys. Rev. C 88, 014904 (2013).

[18] A. Adare et al. (PHENIX Collaboration), Phys. Rev. Lett. 107, 252301 (2011).

[19] K. Aamodt et al. (ALICE Collaboration), Phys. Rev. Lett. 107, 032301 (2011).

[20] S. Chatrchyan et al. (CMS Collaboration), Eur. Phys. J. C 72, 2012 (2012).

[21] B. Alver and G. Roland, Phys. Rev. C 81, 054905 (2010).

[22] P. Sorensen, J. Phys. G 37, 094011 (2010).

[23] M. Alvioli and M. Strikman, Phys. Rev. C 83, 044905 (2011).

[24] D. Teaney and L. Yan, Phys. Rev. C 83, 064904 (2011).

[25] M. Luzum and J.-Y. Ollitrault, Phys. Rev. Lett. 106, 102301 (2011).

[26] F. G. Gardim, F. Grassi, Y. Hama, M. Luzum, and J.-Y. Ollitrault, Phys. Rev. C 83, 064901 (2011).

[27] E. Retinskaya, M. Luzum, and J.-Y. Ollitrault, Phys. Rev. Lett. 108, 252302 (2012).

[28] J. Barrette et al. (E877 Collaboration), Phys. Rev. C 56, 3254 (1997).

[29] C. Alt et al. (NA49 Collaboration), Phys. Rev. C 68, 034903 (2003).

[30] M. Aggarwal et al. (WA98 Collaboration), arXiv:nucl-ex/ 9807004.

[31] J. Brachmann et al., Phys. Rev. C 61, 024909 (2000).

[32] L. Csernai and D. Röhrich, Phys. Lett. B 458, 454 (1999).
[33] J. Adams et al. (STAR Collaboration), Phys. Rev. C 73, 034903 (2006).

[34] B. Abelev et al. (STAR Collaboration), Phys. Rev. Lett. 101, 252301 (2008).

[35] B. Back et al. (PHOBOS Collaboration), Phys. Rev. Lett. 97, 012301 (2006).

[36] G. Agakishiev et al. (STAR Collaboration), Phys. Rev. C 85, 014901 (2012).

[37] R. J. M. Snellings, H. Sorge, S. A. Voloshin, F. Q. Wang, and N. Xu, Phys. Rev. Lett. 84, 2803 (2000).

[38] P. Bozek and I. Wyskiel, Phys. Rev. C 81, 054902 (2010).

[39] L.P. Csernai, V.K. Magas, H. Stocker, and D. D. Strottman, Phys. Rev. C 84, 024914 (2011).

[40] J. Bleibel, G. Burau, and C. Fuchs, Phys. Lett. B 659, 520 (2008).

[41] H. Agakishiev et al. (STAR Collaboration), arXiv:1010.0690.

[42] K. Aamodt et al. (ALICE Collaboration), Phys. Lett. B 708, 249 (2012).

[43] B. Abelev et al. (ALICE Collaboration), Phys. Rev. Lett. 110, 012301 (2013).

[44] K. Fukushima, D. E. Kharzeev, and H. J. Warringa, Phys. Rev. D 78, 074033 (2008).

[45] K. Aamodt et al. (ALICE Collaboration), JINST 3, S08002 (2008).

[46] J. Alme et al., Nucl. Instrum. Methods Phys. Res., Sect. A 622, 316 (2010).

[47] F. Carminati et al. (ALICE Collaboration), J. Phys. G 30, 1517 (2004).

[48] B. Abelev et al. (ALICE Collaboration) Phys. Rev. C 88, 044909 (2013).

[49] B. Abelev et al. (ALICE Collaboration), Phys. Rev. Lett. 109, 252302 (2012).

[50] I. Selyuzhenkov and S. Voloshin, Phys. Rev. C 77, 034904 (2008).

[51] B. Abelev et al. (ALICE Collaboration), http:// hepdata.cedar.ac.uk/view/ins1238980.

[52] I. Selyuzhenkov (ALICE Collaboration), J. Phys. G 38, 124167 (2011).

[53] U. W. Heinz and P. F. Kolb, J. Phys. G 30, S1229 (2004).

B. Abelev, ${ }^{1}$ J. Adam,${ }^{2}$ D. Adamová, ${ }^{3}$ A. M. Adare,${ }^{4}$ M. M. Aggarwal,${ }^{5}$ G. Aglieri Rinella, ${ }^{6}$ M. Agnello,, 8 A. G. Agocs, ${ }^{9}$ A. Agostinelli, ${ }^{10}$ Z. Ahammed, ${ }^{11}$ N. Ahmad, ${ }^{12}$ A. Ahmad Masoodi, ${ }^{12}$ I. Ahmed, ${ }^{13}$ S. A. Ahn, ${ }^{14}$ S. U. Ahn, ${ }^{14}$ I. Aimo, ${ }^{15,7,8}$ M. Ajaz, ${ }^{13}$ A. Akindinov ${ }^{16}$ D. Aleksandrov,${ }^{17}$ B. Alessandro, ${ }^{7}$ D. Alexandre,${ }^{18}$ A. Alici, ${ }^{19,20}$ A. Alkin, ${ }^{21} \mathrm{~J}$. Alme,${ }^{22}$ T. Alt,${ }^{23} \mathrm{~V}$. Altini, ${ }^{24} \mathrm{~S}$. Altinpinar, ${ }^{25}$ I. Altsybeev, ${ }^{26} \mathrm{C}$. Andrei, ${ }^{27}$ A. Andronic, ${ }^{28}$ V. Anguelov, ${ }^{29}$ J. Anielski, ${ }^{30}$ C. Anson, ${ }^{31}$ T. Antičici,${ }^{32}$ F. Antinori, ${ }^{33}$ P. Antonioli, ${ }^{19}$ L. Aphecetche,${ }^{34}$ H. Appelshäuser, ${ }^{35}$ N. Arbor, ${ }^{36}$ S. Arcelli, ${ }^{10}$ A. Arend, ${ }^{35}$ N. Armesto, ${ }^{37}$ R. Arnaldi, ${ }^{7}$ T. Aronsson, ${ }^{4}$ I. C. Arsene, ${ }^{28}$ M. Arslandok, ${ }^{35}$ A. Asryan, ${ }^{26}$ A. Augustinus,${ }^{6}$ R. Averbeck,${ }^{28}$ T. C. Awes,${ }^{38}$ J. Äystö, ${ }^{39}$ M. D. Azmi, ${ }^{12,40}$ M. Bach, ${ }^{23}$ A. Badalà, ${ }^{41}$ Y. W. Baek, ${ }^{42,43}$ R. Bailhache,${ }^{35}$ R. Bala ${ }^{44,7}$ A. Baldisseri, ${ }^{45}$ F. Baltasar Dos Santos Pedrosa, ${ }^{6}$ J. Bán, ${ }^{46}$ R. C. Baral, ${ }^{47}$ R. Barbera, ${ }^{48}$ F. Barile, ${ }^{24}$ G. G. Barnaföldi, ${ }^{9}$ L. S. Barnby,${ }^{18}$ V. Barret,${ }^{42}$ J. Bartke, ${ }^{49}$ M. Basile,${ }^{10}$ N. Bastid ${ }^{42}$ S. Basu, ${ }^{11}$ B. Bathen, ${ }^{30}$ G. Batigne, ${ }^{34}$ B. Batyunya,${ }^{50}$ P. C. Batzing,${ }^{51}$ C. Baumann,${ }^{35}$ I. G. Bearden, ${ }^{52}$ H. Beck, ${ }^{35}$ N. K. Behera ${ }^{53}$ I. Belikov ${ }^{54}$ F. Bellini, ${ }^{10}$ R. Bellwied, ${ }^{55}$ E. Belmont-Moreno, ${ }^{56}$ G. Bencedi, ${ }^{9}$ S. Beole ${ }^{15}$ I. Berceanu, ${ }^{27}$ A. Bercuci, ${ }^{27}$ Y. Berdnikov, ${ }^{57}$ D. Berenyi, ${ }^{9}$ A. A. E. Bergognon, ${ }^{34}$ R. A. Bertens, ${ }^{58}$ D. Berzano, ${ }^{15,7}$ L. Betev, ${ }^{6}$ A. Bhasin, ${ }^{44}$ A. K. Bhati, ${ }^{5}$ J. Bhom,${ }^{59}$ L. Bianchi, ${ }^{15}$ N. Bianchi, ${ }^{60}$ C. Bianchin,${ }^{58}$ J. Bielčík, ${ }^{2}$ J. Bielč́́ková, ${ }^{3}$ A. Bilandzic ${ }^{52}$ S. Bjelogrlic, ${ }^{58}$ F. Blanco,${ }^{55}$ F. Blanco,${ }^{61}$ D. Blau, ${ }^{17}$ C. Blume,${ }^{35}$ M. Boccioli, ${ }^{6}$ F. Bock,${ }^{62,63}$ S. Böttger, ${ }^{64}$ A. Bogdanov, ${ }^{65} \mathrm{H}$. Bøggild, ${ }^{52} \mathrm{M}$. Bogolyubsky, ${ }^{66}$ L. Boldizsár, ${ }^{9}$ M. Bombara, ${ }^{67} \mathrm{~J}$. Book, ${ }^{35} \mathrm{H}$. Borel, ${ }^{45}$ A. Borissov, ${ }^{68}$ F. Bossú, ${ }^{40}$ M. Botje, ${ }^{69}$ E. Botta, ${ }^{15}$ E. Braidot, ${ }^{63}$ P. Braun-Munzinger, ${ }^{28}$ M. Bregant,${ }^{34}$ T. Breitner,${ }^{64}$ 
T. A. Broker, ${ }^{35}$ T. A. Browning, ${ }^{70}$ M. Broz, ${ }^{71}$ R. Brun, ${ }^{6}$ E. Bruna, ${ }^{15,7}$ G. E. Bruno,${ }^{24}$ D. Budnikov, ${ }^{72}$ H. Buesching, ${ }^{35}$ S. Bufalino, ${ }^{15,7}$ P. Buncic, ${ }^{6}$ O. Busch, ${ }^{29}$ Z. Buthelezi, ${ }^{40}$ D. Caffarri, ${ }^{73,33}$ X. Cai,${ }^{74}$ H. Caines, ${ }^{4}$ A. Caliva,${ }^{58}$ E. Calvo Villar, ${ }^{75}$ P. Camerini, ${ }^{76}$ V. Canoa Roman, ${ }^{77}$ G. Cara Romeo, ${ }^{19}$ F. Carena, ${ }^{6}$ W. Carena, ${ }^{6}$ N. Carlin Filho, ${ }^{78}$ F. Carminati, ${ }^{6}$ A. Casanova Díaz, ${ }^{60}$ J. Castillo Castellanos, ${ }^{45}$ J. F. Castillo Hernandez, ${ }^{28}$ E. A. R. Casula ${ }^{79}$ V. Catanescu, ${ }^{27}$ C. Cavicchioli, ${ }^{6}$ C. Ceballos Sanchez, ${ }^{80}$ J. Cepila, ${ }^{2}$ P. Cerello, ${ }^{7}$ B. Chang, ${ }^{39,81}$ S. Chapeland, ${ }^{6}$ J. L. Charvet, ${ }^{45}$ S. Chattopadhyay, ${ }^{11}$ S. Chattopadhyay, ${ }^{82}$ M. Cherney ${ }^{83}$ C. Cheshkov, ${ }^{6,84}$ B. Cheynis, ${ }^{84}$ V. Chibante Barroso, ${ }^{6}$ D. D. Chinellato, ${ }^{55}$ P. Chochula, ${ }^{6}$ M. Chojnacki, ${ }^{52}$ S. Choudhury, ${ }^{11}$ P. Christakoglou, ${ }^{69}$ C. H. Christensen, ${ }^{52}$ P. Christiansen, ${ }^{85}$ T. Chujo, ${ }^{59}$ S. U. Chung, ${ }^{86}$ C. Cicalo, ${ }^{87}$ L. Cifarelli, ${ }^{10,20}$ F. Cindolo, ${ }^{19}$ J. Cleymans, ${ }^{40}$ F. Colamaria, ${ }^{24}$ D. Colella, ${ }^{24}$ A. Collu, ${ }^{79}$ G. Conesa Balbastre, ${ }^{36}$ Z. Conesa del Valle, ${ }^{6,88}$ M. E. Connors, ${ }^{4}$ G. Contin, ${ }^{76}$ J. G. Contreras, ${ }^{77}$ T. M. Cormier,${ }^{68}$ Y. Corrales Morales, ${ }^{15}$ P. Cortese, ${ }^{89}$ I. Cortés Maldonado, ${ }^{90}$ M. R. Cosentino, ${ }^{63}$ F. Costa, ${ }^{6}$ M. E. Cotallo, ${ }^{61}$ E. Crescio, ${ }^{77}$ P. Crochet, ${ }^{42}$ E. Cruz Alaniz, ${ }^{56}$ R. Cruz Albino, ${ }^{77}$ E. Cuautle, ${ }^{91}$ L. Cunqueiro,${ }^{60}$ T. R. Czopowicz, ${ }^{92}$ A. Dainese,${ }^{73,33}$ R. Dang,${ }^{74}$ A. Danu, ${ }^{93}$ D. Das,${ }^{82}$ I. Das, ${ }^{88}$ S. Das, ${ }^{94}$ K. Das, ${ }^{82}$ A. Dash, ${ }^{95}$ S. Dash,${ }^{53}$ S. De, ${ }^{11}$ G. O. V. de Barros, ${ }^{78}$ A. De Caro, ${ }^{96,20}$ G. de Cataldo, ${ }^{97}$ J. de Cuveland, ${ }^{23}$ A. De Falco, ${ }^{79}$ D. De Gruttola,${ }^{96,20}$ H. Delagrange, ${ }^{34}$ A. Deloff, ${ }^{98}$ N. De Marco, ${ }^{7}$ E. Dénes, ${ }^{9}$ S. De Pasquale, ${ }^{96}$ A. Deppman, ${ }^{78}$ G. D'Erasmo, ${ }^{24}$ R. de Rooij, ${ }^{58}$ M. A. Diaz Corchero, ${ }^{61}$ D. Di Bari, ${ }^{24}$ T. Dietel, ${ }^{30}$

C. Di Giglio, ${ }^{24}$ S. Di Liberto, ${ }^{99}$ A. Di Mauro, ${ }^{6}$ P. Di Nezza, ${ }^{60}$ R. Divià, ${ }^{6} \varnothing$. Djuvsland, ${ }^{25}$ A. Dobrin,${ }^{68,85,58}$

T. Dobrowolski, ${ }^{98}$ B. Dönigus, ${ }^{28,35}$ O. Dordic,${ }^{51}$ A. K. Dubey, ${ }^{11}$ A. Dubla,${ }^{58}$ L. Ducroux,${ }^{84}$ P. Dupieux, ${ }^{42}$ A. K. Dutta Majumdar, ${ }^{82}$ D. Elia, ${ }^{97}$ B. G. Elwood, ${ }^{100}$ D. Emschermann, ${ }^{30}$ H. Engel,${ }^{64}$ B. Erazmus, ${ }^{6,34}$ H. A. Erdal, ${ }^{22}$ D. Eschweiler, ${ }^{23}$ B. Espagnon, ${ }^{88}$ M. Estienne, ${ }^{34}$ S. Esumi,${ }^{59}$ D. Evans, ${ }^{18}$ S. Evdokimov, ${ }^{66}$ G. Eyyubova,${ }^{51}$ D. Fabris, ${ }^{73,33}$ J. Faivre, ${ }^{36}$ D. Falchieri, ${ }^{10}$ A. Fantoni, ${ }^{60}$ M. Fasel, ${ }^{29}$ D. Fehlker, ${ }^{25}$ L. Feldkamp, ${ }^{30}$ D. Felea, ${ }^{93}$ A. Feliciello, ${ }^{7}$ B. Fenton-Olsen, ${ }^{63}$ G. Feofilov, ${ }^{26}$ A. Fernández Téllez,${ }^{90}$ A. Ferretti, ${ }^{15}$ A. Festanti, ${ }^{73}$ J. Figiel, ${ }^{49}$ M. A. S. Figueredo,${ }^{78}$ S. Filchagin, ${ }^{72}$ D. Finogeev, ${ }^{101}$ F. M. Fionda, ${ }^{24}$ E. M. Fiore,${ }^{24}$ E. Floratos, ${ }^{102}$ M. Floris, ${ }^{6}$ S. Foertsch, ${ }^{40}$ P. Foka, ${ }^{28}$ S. Fokin, ${ }^{17}$ E. Fragiacomo, ${ }^{103}$ A. Francescon, ${ }^{6,73}$ U. Frankenfeld, ${ }^{28}$ U. Fuchs,${ }^{6}$ C. Furget, ${ }^{36}$ M. Fusco Girard ${ }^{96}$ J. J. Gaardhøje, ${ }^{52}$ M. Gagliardi, ${ }^{15}$ A. Gago, ${ }^{75}$ M. Gallio, ${ }^{15}$ D. R. Gangadharan, ${ }^{31}$ P. Ganoti, ${ }^{38}$ C. Garabatos, ${ }^{28}$ E. Garcia-Solis, ${ }^{100}$ C. Gargiulo, ${ }^{6}$ I. Garishvili, ${ }^{1}$ J. Gerhard, ${ }^{23}$ M. Germain, ${ }^{34}$ A. Gheata, ${ }^{6}$ M. Gheata ${ }^{93,6}$ B. Ghidini ${ }^{24}$ P. Ghosh,${ }^{11}$ P. Gianotti, ${ }^{60}$ P. Giubellino, ${ }^{6}$ E. Gladysz-Dziadus ${ }^{49}$ P. Glässel, ${ }^{29}$ L. Goerlich, ${ }^{49}$ R. Gomez, ${ }^{104,77}$ E. G. Ferreiro, ${ }^{37}$ P. González-Zamora, ${ }^{61}$ S. Gorbunov, ${ }^{23}$ A. Goswami, ${ }^{105}$ S. Gotovac, ${ }^{106}$ L. K. Graczykowski, ${ }^{92}$ R. Grajcarek, ${ }^{29}$ A. Grelli, ${ }^{58}$ A. Grigoras, ${ }^{6}$ C. Grigoras,${ }^{6}$ V. Grigoriev, ${ }^{65}$ A. Grigoryan, ${ }^{107}$ S. Grigoryan, ${ }^{50}$ B. Grinyov, ${ }^{21}$ N. Grion, ${ }^{103}$ P. Gros, ${ }^{85}$ J. F. Grosse-Oetringhaus, ${ }^{6}$ J.-Y. Grossiord ${ }^{84}$ R. Grosso, ${ }^{6}$ F. Guber, ${ }^{101}$ R. Guernane, ${ }^{36}$ B. Guerzoni, ${ }^{10}$ M. Guilbaud, ${ }^{84}$ K. Gulbrandsen,${ }^{52}$ H. Gulkanyan, ${ }^{107}$ T. Gunji, ${ }^{108}$ A. Gupta, ${ }^{44}$ R. Gupta, ${ }^{44}$ R. Haake, ${ }^{30}$ Ø. Haaland,${ }^{25}$ C. Hadjidakis, ${ }^{88}$ M. Haiduc, ${ }^{93}$ H. Hamagaki, ${ }^{108}$ G. Hamar, ${ }^{9}$ B. H. Han, ${ }^{109}$ L. D. Hanratty, ${ }^{18}$ A. Hansen, ${ }^{52}$ J. W. Harris, ${ }^{4}$ A. Harton, ${ }^{100}$ D. Hatzifotiadou, ${ }^{19}$ S. Hayashi, ${ }^{108}$ A. Hayrapetyan, ${ }^{6,107}$ S. T. Heckel, ${ }^{35}$ M. Heide, ${ }^{30}$ H. Helstrup,${ }^{22}$ A. Herghelegiu, ${ }^{27}$ G. Herrera Corral, ${ }^{77}$ N. Herrmann, ${ }^{29}$ B. A. Hess, ${ }^{110}$ K. F. Hetland ${ }^{22}$ B. Hicks, ${ }^{4}$ B. Hippolyte, ${ }^{54}$ Y. Hori, ${ }^{108}$ P. Hristov,${ }^{6}$ I. Hřivnáčová, ${ }^{88}$ M. Huang, ${ }^{25}$ T. J. Humanic, ${ }^{31}$ D. S. Hwang, ${ }^{109}$ R. Ichou, ${ }^{42}$ R. Ilkaev,${ }^{72}$ I. Ilkiv, ${ }^{98}$ M. Inaba, ${ }^{59}$ E. Incani, ${ }^{79}$ P. G. Innocenti, ${ }^{6}$ G. M. Innocenti, ${ }^{15}$ C. Ionita, ${ }^{6}$ M. Ippolitov, ${ }^{17}$ M. Irfan, ${ }^{12}$ V. Ivanov, ${ }^{57}$ M. Ivanov, ${ }^{28}$ A. Ivanov, ${ }^{26}$ O. Ivanytskyi, ${ }^{21}$ A. Jachołkowski, ${ }^{48}$ P. M. Jacobs, ${ }^{63}$ C. Jahnke, ${ }^{78}$ H. J. Jang,,${ }^{14}$ M. A. Janik, ${ }^{92}$ P. H. S. Y. Jayarathna,${ }^{55}$ S. Jena, ${ }^{53}$ D. M. Jha ${ }^{68}$ R. T. Jimenez Bustamante, ${ }^{91}$ P. G. Jones, ${ }^{18}$ H. Jung, ${ }^{43}$ A. Jusko, ${ }^{18}$ A. B. Kaidalov, ${ }^{16}$ S. Kalcher, ${ }^{23}$ P. Kaliňák, ${ }^{46}$ T. Kalliokoski ${ }^{39}$ A. Kalweit, ${ }^{6}$ J. H. Kang, ${ }^{81}$ V. Kaplin, ${ }^{65}$ S. Kar, ${ }^{11}$ A. Karasu Uysal, ${ }^{111}$ O. Karavichev, ${ }^{101}$ T. Karavicheva, ${ }^{101}$ E. Karpechev, ${ }^{101}$ A. Kazantsev, ${ }^{17}$ U. Kebschull, ${ }^{64}$ R. Keidel, ${ }^{112}$ B. Ketzer, ${ }^{35,113}$ M. M. Khan, ${ }^{12}$ P. Khan, ${ }^{82}$ K. H. Khan, ${ }^{13}$ S. A. Khan, ${ }^{11}$ A. Khanzadeev ${ }^{57}$ Y. Kharlov, ${ }^{66}$ B. Kileng, ${ }^{22}$ J. S. Kim, ${ }^{43}$

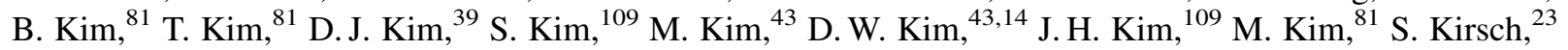
I. Kisel, ${ }^{23}$ S. Kiselev, ${ }^{16}$ A. Kisiel, ${ }^{92}$ G. Kiss, ${ }^{9}$ J. L. Klay, ${ }^{114}$ J. Klein, ${ }^{29}$ C. Klein-Bösing, ${ }^{30}$ M. Kliemant, ${ }^{35}$ A. Kluge, ${ }^{6}$ M. L. Knichel, ${ }^{28}$ A. G. Knospe, ${ }^{115}$ M. K. Köhler ${ }^{28}$ T. Kollegger ${ }^{23}$ A. Kolojvari, ${ }^{26}$ M. Kompaniets, ${ }^{26}$ V. Kondratiev, ${ }^{26}$ N. Kondratyeva, ${ }^{65}$ A. Konevskikh, ${ }^{101}$ V. Kovalenko, ${ }^{26}$ M. Kowalski, ${ }^{49}$ S. Kox,${ }^{36}$ G. Koyithatta Meethaleveedu, ${ }^{53}$ J. Kral, ${ }^{39}$ I. Králik, ${ }^{46}$ F. Kramer, ${ }^{35}$ A. Kravčáková, ${ }^{67}$ M. Krelina, ${ }^{2}$ M. Kretz, ${ }^{23}$ M. Krivda, ${ }^{18,46}$ F. Krizek, ${ }^{39,2,3}$ M. Krus, ${ }^{2}$ E. Kryshen, ${ }^{57}$ M. Krzewicki, ${ }^{28}$ V. Kucera, ${ }^{3}$ Y. Kucheriaev, ${ }^{17}$ T. Kugathasan, ${ }^{6}$ C. Kuhn, ${ }^{54}$ P. G. Kuijer, ${ }^{69}$ I. Kulakov, ${ }^{35}$ J. Kumar, ${ }^{53}$ P. Kurashvili, ${ }^{98}$ A. Kurepin, ${ }^{101}$ A. B. Kurepin, ${ }^{101}$ A. Kuryakin, ${ }^{72}$ S. Kushpil, ${ }^{3}$ V. Kushpil, ${ }^{3}$

H. Kvaerno, ${ }^{51}$ M. J. Kweon, ${ }^{29}$ Y. Kwon, ${ }^{81}$ P. Ladrón de Guevara, ${ }^{91}$ C. Lagana Fernandes, ${ }^{78}$ I. Lakomov, ${ }^{88}$ R. Langoy, ${ }^{16}$ S. L. La Pointe, ${ }^{58}$ C. Lara, ${ }^{64}$ A. Lardeux ${ }^{34}$ P. La Rocca, ${ }^{48}$ R. Lea,${ }^{76}$ M. Lechman, ${ }^{6}$ G. R. Lee, ${ }^{18}$ S. C. Lee, ${ }^{43}$ I. Legrand, ${ }^{6}$ J. Lehnert, ${ }^{35}$ R. C. Lemmon, ${ }^{117}$ M. Lenhardt,${ }^{28}$ V. Lenti, ${ }^{97}$ H. León, ${ }^{56}$ M. Leoncino, ${ }^{15}$ 
I. León Monzón, ${ }^{104}$ P. Lévai, ${ }^{9}$ S. Li,${ }^{42,74}$ J. Lien, ${ }^{25,116}$ R. Lietava, ${ }^{18}$ S. Lindal,${ }^{51}$ V. Lindenstruth, ${ }^{23}$ C. Lippmann, ${ }^{28,6}$ M. A. Lisa, ${ }^{31}$ H. M. Ljunggren,${ }^{85}$ D. F. Lodato,${ }^{58}$ P. I. Loenne, ${ }^{25}$ V. R. Loggins, ${ }^{68}$ V. Loginov, ${ }^{65}$ D. Lohner, ${ }^{29}$ C. Loizides, ${ }^{63}$ K. K. Loo,${ }^{39}$ X. Lopez, ${ }^{42}$ E. López Torres, ${ }^{80}$ G. Løvhøiden,${ }^{51}$ X.-G. Lu, ${ }^{29}$ P. Luettig, ${ }^{35}$ M. Lunardon, ${ }^{73}$ J. Luo,${ }^{74}$ G. Luparello, ${ }^{58}$ C. Luzzi, ${ }^{6}$ R. Ma,${ }^{4}$ K. Ma,${ }^{74}$ D. M. Madagodahettige-Don, ${ }^{55}$ A. Maevskaya,${ }^{101}$ M. Mager, ${ }^{118,6}$ D. P. Mahapatra, ${ }^{47}$ A. Maire, ${ }^{29}$ M. Malaev,${ }^{57}$ I. Maldonado Cervantes, ${ }^{91}$ L. Malinina, ${ }^{50,119}$ D. Mal'Kevich, ${ }^{16}$ P. Malzacher, ${ }^{28}$ A. Mamonov, ${ }^{72}$ L. Manceau, ${ }^{7}$ L. Mangotra, ${ }^{44}$ V. Manko, ${ }^{17}$ F. Manso, ${ }^{42}$ V. Manzari, ${ }^{97}$ M. Marchisone, ${ }^{42,15}$ J. Mareš, ${ }^{120}$ G. V. Margagliotti, ${ }^{76,103}$ A. Margotti, ${ }^{19}$ A. Marín, ${ }^{28}$ C. Markert,,${ }^{6,115}$ M. Marquard, ${ }^{35}$ I. Martashvili, ${ }^{121}$ N. A. Martin, ${ }^{28}$ J. Martin Blanco, ${ }^{34}$ P. Martinengo, ${ }^{6}$ M. I. Martínez, ${ }^{90}$ G. Martínez García ${ }^{34}$ Y. Martynov, ${ }^{21}$ A. Mas,${ }^{34}$ S. Masciocchi, ${ }^{28}$ M. Masera, ${ }^{15}$ A. Masoni, ${ }^{87}$ L. Massacrier,${ }^{34}$ A. Mastroserio, ${ }^{24}$ A. Matyja ${ }^{49}$ C. Mayer ${ }^{49}$ J. Mazer,${ }^{121}$ R. Mazumder, ${ }^{122}$ M. A. Mazzoni, ${ }^{99}$ F. Meddi,${ }^{123}$ A. Menchaca-Rocha, ${ }^{56}$ J. Mercado Pérez, ${ }^{29}$ M. Meres,${ }^{71}$ Y. Miake, ${ }^{59}$ K. Mikhaylov, ${ }^{50,16}$ L. Milano, ${ }^{6,15}$ J. Milosevic, ${ }^{51,124}$ A. Mischke, ${ }^{58}$ A. N. Mishra, ${ }^{105,122}$ D. Miśkowiec, ${ }^{28}$ C. Mitu, ${ }^{93}$ J. Mlynarz, ${ }^{68}$ B. Mohanty, ${ }^{11,125}$ L. Molnar, ${ }^{9,54}$ L. Montaño Zetina, ${ }^{77}$ M. Monteno, ${ }^{7}$ E. Montes, ${ }^{61}$ T. Moon, ${ }^{81}$ M. Morando,${ }^{73}$

D. A. Moreira De Godoy ${ }^{78}$ S. Moretto, ${ }^{73}$ A. Morreale ${ }^{39}$ A. Morsch, ${ }^{6}$ V. Muccifora, ${ }^{60}$ E. Mudnic, ${ }^{106}$ S. Muhuri, ${ }^{11}$ M. Mukherjee, ${ }^{11}$ H. Müller, ${ }^{6}$ M. G. Munhoz, ${ }^{78}$ S. Murray,${ }^{40}$ L. Musa, ${ }^{6}$ J. Musinsky, ${ }^{46}$ B. K. Nandi, ${ }^{53}$ R. Nania, ${ }^{19}$ E. Nappi,${ }^{97}$ M. Nasar, ${ }^{126}$ C. Nattrass, ${ }^{121}$ T. K. Nayak, ${ }^{11}$ S. Nazarenko, ${ }^{72}$ A. Nedosekin,${ }^{16}$ M. Nicassio,,${ }^{24,28}$ M. Niculescu, ${ }^{93,6}$ B. S. Nielsen, ${ }^{52}$ S. Nikolaev, ${ }^{17}$ V. Nikolic, ${ }^{32}$ V. Nikulin, ${ }^{57}$ S. Nikulin, ${ }^{17}$ B. S. Nilsen, ${ }^{83}$ M. S. Nilsson, ${ }^{51}$ F. Noferini, ${ }^{19,20}$ P. Nomokonov, ${ }^{50}$ G. Nooren,${ }^{58}$ A. Nyanin, ${ }^{17}$ A. Nyatha, ${ }^{53}$ C. Nygaard, ${ }^{52}$ J. Nystrand, ${ }^{25}$ A. Ochirov, ${ }^{26}$ H. Oeschler, ${ }^{18,6,29}$ S. K. Oh, ${ }^{43}$ S. Oh, ${ }^{4}$ L. Olah, ${ }^{9}$ J. Oleniacz, ${ }^{92}$ A. C. Oliveira Da Silva, ${ }^{78}$ J. Onderwaater, ${ }^{28}$ C. Oppedisano, ${ }^{7}$ A. Ortiz Velasquez, ${ }^{85,91}$ A. Oskarsson, ${ }^{85}$ P. Ostrowski, ${ }^{92}$ J. Otwinowski, ${ }^{28}$ K. Oyama, ${ }^{29}$ K. Ozawa, ${ }^{108}$ Y. Pachmayer, ${ }^{29}$ M. Pachr, ${ }^{2}$ F. Padilla, ${ }^{15}$ P. Pagano, ${ }^{96}$ G. Paic,${ }^{91}$ F. Painke, ${ }^{23}$ C. Pajares, ${ }^{37}$ S. K. Pal, ${ }^{11}$ A. Palaha ${ }^{18}$ A. Palmeri, ${ }^{41}$ V. Papikyan, ${ }^{107}$ G. S. Pappalardo,${ }^{41}$ W. J. Park ${ }^{28}$ A. Passfeld,${ }^{30}$ D. I. Patalakha, ${ }^{66}$ V. Paticchio, ${ }^{97}$ B. Paul,${ }^{82}$ A. Pavlinov, ${ }^{68}$ T. Pawlak,${ }^{92}$ T. Peitzmann,${ }^{58}$ H. Pereira Da Costa, ${ }^{45}$

E. Pereira De Oliveira Filho, ${ }^{78}$ D. Peresunko, ${ }^{17}$ C. E. Pérez Lara, ${ }^{69}$ D. Perrino,${ }^{24}$ W. Peryt, ${ }^{92, *}$ A. Pesci, ${ }^{19}$ Y. Pestov, ${ }^{127}$ V. Petráček, ${ }^{2}$ M. Petran, ${ }^{2}$ M. Petris,${ }^{27}$ P. Petrov, ${ }^{18}$ M. Petrovici,${ }^{27}$ C. Petta, ${ }^{48}$ S. Piano, ${ }^{103}$ M. Pikna,${ }^{71}$ P. Pillot, ${ }^{34}$ O. Pinazza, ${ }^{6}$ L. Pinsky, ${ }^{55}$ N. Pitz, ${ }^{35}$ D. B. Piyarathna, ${ }^{55}$ M. Planinic,${ }^{32}$ M. Płoskon,,${ }^{63}$ J. Pluta, ${ }^{92}$

T. Pocheptsov, ${ }^{50}$ S. Pochybova, ${ }^{9}$ P. L. M. Podesta-Lerma, ${ }^{104}$ M. G. Poghosyan, ${ }^{6}$ K. Polák, ${ }^{120}$ B. Polichtchouk, ${ }^{66}$ N. Poljak, ${ }^{58,32}$ A. Pop, ${ }^{27}$ S. Porteboeuf-Houssais, ${ }^{42}$ V. Pospíšil,${ }^{2}$ B. Potukuchi, ${ }^{44}$ S. K. Prasad,${ }^{68}$ R. Preghenella, ${ }^{19,20}$ F. Prino, ${ }^{7}$ C. A. Pruneau ${ }^{68}$ I. Pshenichnov,${ }^{101}$ G. Puddu, ${ }^{79}$ V. Punin, ${ }^{72}$ J. Putschke,${ }^{68}$ H. Qvigstad,${ }^{51}$ A. Rachevski, ${ }^{103}$ A. Rademakers, ${ }^{6}$ J. Rak,${ }^{39}$ A. Rakotozafindrabe, ${ }^{45}$ L. Ramello, ${ }^{89}$ S. Raniwala, ${ }^{105}$ R. Raniwala, ${ }^{105}$ S. S. Räsänen, ${ }^{39}$ B. T. Rascanu, ${ }^{35}$ D. Rathee, ${ }^{5}$ W. Rauch, ${ }^{6}$ A. W. Rauf, ${ }^{13}$ V. Razazi,${ }^{79}$ K. F. Read ${ }^{121}$ J. S. Real, ${ }^{36}$ K. Redlich,${ }^{98,128}$ R. J. Reed, ${ }^{4}$ A. Rehman, ${ }^{25}$ P. Reichelt,${ }^{35}$ M. Reicher, ${ }^{58}$ F. Reidt, ${ }^{29}$ R. Renfordt,${ }^{35}$ A. R. Reolon, ${ }^{60}$ A. Reshetin, ${ }^{101}$ F. Rettig, ${ }^{23}$ J.-P. Revol, ${ }^{6}$ K. Reygers,${ }^{29}$ L. Riccati, ${ }^{7}$ R. A. Ricci, ${ }^{129}$ T. Richert, ${ }^{85}$ M. Richter,${ }^{51}$ P. Riedler, ${ }^{6}$ W. Riegler, ${ }^{6}$ F. Riggi, ${ }^{48,41}$ A. Rivetti, ${ }^{7}$ M. Rodríguez Cahuantzi, ${ }^{90}$ A. Rodriguez Manso, ${ }^{69} \mathrm{~K}$. Røed,${ }^{25,51}$ E. Rogochaya,${ }^{50}$ D. Rohr, ${ }^{23}$ D. Röhrich, ${ }^{25}$ R. Romita, ${ }^{28,117}$ F. Ronchetti, ${ }^{60}$ P. Rosnet, ${ }^{42}$ S. Rossegger, ${ }^{6}$ A. Rossi, ${ }^{6}$ P. Roy, ${ }^{82}$ C. Roy, ${ }^{54}$ A. J. Rubio Montero, ${ }^{61}$ R. Rui,${ }^{76}$ R. Russo, ${ }^{15}$ E. Ryabinkin, ${ }^{17}$ A. Rybicki, ${ }^{49}$ S. Sadovsky,${ }^{66}$ K. Šafař́k, ${ }^{6}$ R. Sahoo, ${ }^{122}$ P. K. Sahu, ${ }^{47}$ J. Saini, ${ }^{11}$ H. Sakaguchi, ${ }^{130}$ S. Sakai,${ }^{63,60}$ D. Sakata, ${ }^{59}$ C. A. Salgado, ${ }^{37}$ J. Salzwedel, ${ }^{31}$ S. Sambyal, ${ }^{44}$ V. Samsonov, ${ }^{57}$ X. Sanchez Castro, ${ }^{54}$ L. Šándor, ${ }^{46}$ A. Sandoval, ${ }^{56}$ M. Sano,${ }^{59}$ G. Santagati, ${ }^{48}$ R. Santoro,,${ }^{6,20}$

D. Sarkar, ${ }^{11}$ E. Scapparone, ${ }^{19}$ F. Scarlassara,${ }^{73}$ R. P. Scharenberg,${ }^{70}$ C. Schiaua,${ }^{27}$ R. Schicker, ${ }^{29}$ C. Schmidt, ${ }^{28}$

H. R. Schmidt, ${ }^{110}$ S. Schuchmann, ${ }^{35}$ J. Schukraft, ${ }^{6}$ M. Schulc, ${ }^{2}$ T. Schuster, ${ }^{4}$ Y. Schutz, ${ }^{6,34}$ K. Schwarz, ${ }^{28}$ K. Schweda, ${ }^{28}$ G. Scioli, ${ }^{10}$ E. Scomparin, ${ }^{7}$ P. A. Scott, ${ }^{18}$ R. Scott,${ }^{121}$ G. Segato, ${ }^{73}$ I. Selyuzhenkov, ${ }^{28}$ S. Senyukov,${ }^{54}$ J. Seo, ${ }^{86}$ S. Serci, ${ }^{79}$ E. Serradilla, ${ }^{61,56}$ A. Sevcenco, ${ }^{93}$ A. Shabetai, ${ }^{34}$ G. Shabratova, ${ }^{50}$ R. Shahoyan,${ }^{6}$ N. Sharma,${ }^{121}$ S. Sharma, ${ }^{44}$ S. Rohni, ${ }^{44}$ K. Shigaki, ${ }^{130}$ K. Shtejer ${ }^{80}$ Y. Sibiriak, ${ }^{17}$ S. Siddhanta, ${ }^{87}$ T. Siemiarczuk, ${ }^{98}$ D. Silvermyr, ${ }^{38}$ C. Silvestre, ${ }^{36}$ G. Simatovic, ${ }^{91,32}$ G. Simonetti, ${ }^{6}$ R. Singaraju, ${ }^{11}$ R. Singh, ${ }^{44}$ S. Singha, ${ }^{11,125}$ V. Singhal, ${ }^{11}$ T. Sinha,${ }^{82}$ B. C. Sinha, ${ }^{11}$ B. Sitar, ${ }^{71}$ M. Sitta, ${ }^{89}$ T. B. Skaali, ${ }^{51}$ K. Skjerdal, ${ }^{25}$ R. Smakal, ${ }^{2}$ N. Smirnov, ${ }^{4}$ R. J. M. Snellings,${ }^{58}$ C. Søgaard,${ }^{85}$ R. Soltz, ${ }^{1}$ J. Song,${ }^{86}$ M. Song, ${ }^{81}$ C. Soos,${ }^{6}$ F. Soramel,${ }^{73}$ M. Spacek, ${ }^{2}$ I. Sputowska, ${ }^{49}$ M. Spyropoulou-Stassinaki, ${ }^{102}$ B. K. Srivastava, ${ }^{70}$ J. Stachel,${ }^{29}$ I. Stan,${ }^{93}$ G. Stefanek,${ }^{98}$ M. Steinpreis,${ }^{31}$ E. Stenlund, ${ }^{85}$ G. Steyn,${ }^{40}$ J. H. Stiller, ${ }^{29}$ D. Stocco, ${ }^{34}$ M. Stolpovskiy, ${ }^{66}$ P. Strmen, ${ }^{71}$ A. A. P. Suaide, ${ }^{78}$ M. A. Subieta Vásquez, ${ }^{15}$ T. Sugitate, ${ }^{130}$ C. Suire,${ }^{88}$ M. Suleymanov,${ }^{13}$ R. Sultanov, ${ }^{16}$ M. Šumbera,${ }^{3}$ T. Susa,${ }^{32}$ T. J. M. Symons, ${ }^{63}$ A. Szanto de Toledo, ${ }^{78}$ I. Szarka, ${ }^{71}$ A. Szczepankiewicz, ${ }^{6}$ M. Szymański,${ }^{92}$ J. Takahashi, ${ }^{95}$ M. A. Tangaro, ${ }^{24}$ J. D. Tapia Takaki,${ }^{88}$ A. Tarantola Peloni,${ }^{35}$ A. Tarazona Martinez, ${ }^{6}$ A. Tauro, ${ }^{6}$ G. Tejeda Muñoz, ${ }^{90}$ 
A. Telesca, ${ }^{6}$ A. Ter Minasyan, ${ }^{17}$ C. Terrevoli, ${ }^{24}$ J. Thäder, ${ }^{28}$ D. Thomas,${ }^{58}$ R. Tieulent,${ }^{84}$ A. R. Timmins,${ }^{55}$ D. Tlusty, ${ }^{2}$ A. Toia,${ }^{23,73,33}$ H. Torii, ${ }^{108}$ L. Toscano, ${ }^{7}$ V. Trubnikov, ${ }^{21}$ D. Truesdale,${ }^{31}$ W. H. Trzaska, ${ }^{39}$ T. Tsuji, ${ }^{108}$ A. Tumkin,${ }^{72}$ R. Turrisi, ${ }^{33}$ T. S. Tveter, ${ }^{51}$ J. Ulery, ${ }^{35}$ K. Ullaland, ${ }^{25}$ J. Ulrich, ${ }^{62,64}$ A. Uras, ${ }^{84}$ G. M. Urciuoli, ${ }^{99}$ G. L. Usai, ${ }^{79}$ M. Vajzer, ${ }^{2,3}$ M. Vala, ${ }^{50,46}$ L. Valencia Palomo, ${ }^{88}$ S. Vallero, ${ }^{15}$ P. Vande Vyvre, ${ }^{6}$ J. W. Van Hoorne, ${ }^{6}$ M. van Leeuwen, ${ }^{58}$ L. Vannucci, ${ }^{129}$ A. Vargas,${ }^{90}$ R. Varma, ${ }^{53}$ M. Vasileiou, ${ }^{102}$ A. Vasiliev, ${ }^{17}$ V. Vechernin, ${ }^{26}$ M. Veldhoen, ${ }^{58} \mathrm{M}$. Venaruzzo ${ }^{76}$ E. Vercellin, ${ }^{15} \mathrm{~S}$. Vergara, ${ }^{90} \mathrm{R}$. Vernet, ${ }^{131} \mathrm{M}$. Verweij, ${ }^{68,58} \mathrm{~L}$. Vickovic, ${ }^{106}$ G. Viesti, ${ }^{73}$ J. Viinikainen, ${ }^{39}$ Z. Vilakazi, ${ }^{40}$ O. Villalobos Baillie, ${ }^{18}$ Y. Vinogradov, ${ }^{72}$ A. Vinogradov, ${ }^{17}$ L. Vinogradov, ${ }^{26}$ T. Virgili, ${ }^{96}$ Y. P. Viyogi, ${ }^{11}$ A. Vodopyanov, ${ }^{50}$ M. A. Völkl ${ }^{29}$ K. Voloshin, ${ }^{16}$ S. Voloshin, ${ }^{68}$ G. Volpe ${ }^{6}$ B. von Haller, ${ }^{6}$ I. Vorobyev ${ }^{26}$ D. Vranic, ${ }^{28,6}$ J. Vrláková, ${ }^{67}$ B. Vulpescu, ${ }^{42}$ A. Vyushin, ${ }^{72}$ B. Wagner, ${ }^{25}$ V. Wagner, ${ }^{2}$ J. Wagner,${ }^{28}$ Y. Wang,${ }^{74}$ M. Wang, ${ }^{74}$ Y. Wang, ${ }^{29}$ D. Watanabe,${ }^{59}$ K. Watanabe,${ }^{59}$ M. Weber,${ }^{55}$ J. P. Wessels,${ }^{30}$ U. Westerhoff,,${ }^{30}$ J. Wiechula, ${ }^{110}$ D. Wielanek, ${ }^{92}$ J. Wikne, ${ }^{51}$ M. Wilde,${ }^{30}$ G. Wilk, ${ }^{98}$ J. Wilkinson,${ }^{29}$ M. C. S. Williams, ${ }^{19}$ M. Winn, ${ }^{29}$ B. Windelband,${ }^{29}$ C. Xiang, ${ }^{74}$ C. G. Yaldo,${ }^{68}$ Y. Yamaguchi, ${ }^{108}$ H. Yang, ${ }^{45,58}$ S. Yang, ${ }^{25}$ P. Yang ${ }^{74}$ S. Yano, ${ }^{130}$ S. Yasnopolskiy, ${ }^{17} \mathrm{~J}$. Yi,${ }^{86} \mathrm{Z}$. Yin, ${ }^{74} \mathrm{I} .-\mathrm{K}$. Yoo, ${ }^{86} \mathrm{~J}$. Yoon, ${ }^{81} \mathrm{I}$. Yushmanov ${ }^{17}$ V. Zaccolo, ${ }^{52}$ C. Zach, ${ }^{2}$ C. Zampolli, ${ }^{19}$ S. Zaporozhets, ${ }^{50}$ A. Zarochentsev, ${ }^{26}$ P. Závada, ${ }^{120}$ N. Zaviyalov, ${ }^{72}$ H. Zbroszczyk, ${ }^{92}$ P. Zelnicek, ${ }^{64}$ I. S. Zgura, ${ }^{93}$ M. Zhalov, ${ }^{57}$ Y. Zhang, ${ }^{74}$ X. Zhang, ${ }^{63,42,74}$ F. Zhang, ${ }^{74}$ H. Zhang ${ }^{74}$ Y. Zhou, ${ }^{58}$ F. Zhou, ${ }^{74}$ D. Zhou, ${ }^{74}$ H. Zhu, ${ }^{74}$ X. Zhu, ${ }^{74}$ J. Zhu, ${ }^{74}$ J. Zhu, ${ }^{74}$ A. Zichichi, ${ }^{10,20}$ A. Zimmermann, ${ }^{29}$ G. Zinovjev, ${ }^{21}$ Y. Zoccarato, ${ }^{84}$ M. Zynovyev, ${ }^{21}$ and M. Zyzak ${ }^{35}$

\section{(ALICE Collaboration)}

${ }^{1}$ Lawrence Livermore National Laboratory, Livermore, California, USA

${ }^{2}$ Faculty of Nuclear Sciences and Physical Engineering, Czech Technical University in Prague, Prague, Czech Republic

${ }^{3}$ Nuclear Physics Institute, Academy of Sciences of the Czech Republic, Řež u Prahy, Czech Republic

${ }^{4}$ Yale University, New Haven, Connecticut, USA

${ }^{5}$ Physics Department, Panjab University, Chandigarh, India

${ }^{6}$ European Organization for Nuclear Research (CERN), Geneva, Switzerland

${ }^{7}$ Sezione INFN, Turin, Italy

${ }^{8}$ Politecnico di Torino, Turin, Italy

${ }^{9}$ Wigner Research Centre for Physics, Hungarian Academy of Sciences, Budapest, Hungary

${ }^{10}$ Dipartimento di Fisica e Astronomia dell'Università and Sezione INFN, Bologna, Italy

${ }^{11}$ Variable Energy Cyclotron Centre, Kolkata, India

${ }^{12}$ Department of Physics Aligarh Muslim University, Aligarh, India

${ }^{13}$ COMSATS Institute of Information Technology (CIIT), Islamabad, Pakistan

${ }^{14}$ Korea Institute of Science and Technology Information, Daejeon, South Korea

${ }^{15}$ Dipartimento di Fisica dell'Università and Sezione INFN, Turin, Italy

${ }^{16}$ Institute for Theoretical and Experimental Physics, Moscow, Russia

${ }^{17}$ Russian Research Centre Kurchatov Institute, Moscow, Russia

${ }^{18}$ School of Physics and Astronomy, University of Birmingham, Birmingham, United Kingdom

${ }^{19}$ Sezione INFN, Bologna, Italy

${ }^{20}$ Centro Fermi-Museo Storico della Fisica e Centro Studi e Ricerche "Enrico Fermi," Rome, Italy

${ }^{21}$ Bogolyubov Institute for Theoretical Physics, Kiev, Ukraine

${ }^{22}$ Faculty of Engineering, Bergen University College, Bergen, Norway

${ }^{23}$ Frankfurt Institute for Advanced Studies, Johann Wolfgang Goethe-Universität Frankfurt, Frankfurt, Germany

${ }^{24}$ Dipartimento Interateneo di Fisica "M. Merlin" and Sezione INFN, Bari, Italy

${ }^{25}$ Department of Physics and Technology, University of Bergen, Bergen, Norway

${ }^{26}$ V. Fock Institute for Physics, St. Petersburg State University, St. Petersburg, Russia

${ }^{27}$ National Institute for Physics and Nuclear Engineering, Bucharest, Romania

${ }^{28}$ Research Division and ExtreMe Matter Institute EMMI, GSI Helmholtzzentrum für Schwerionenforschung, Darmstadt, Germany

${ }^{29}$ Physikalisches Institut, Ruprecht-Karls-Universität Heidelberg, Heidelberg, Germany

${ }^{30}$ Institut für Kernphysik, Westfälische Wilhelms-Universität Münster, Münster, Germany

${ }^{31}$ Department of Physics, The Ohio State University, Columbus, Ohio, USA

${ }^{32}$ Rudjer Bošković Institute, Zagreb, Croatia

${ }^{33}$ Sezione INFN, Padova, Italy

${ }^{34}$ SUBATECH, Ecole des Mines de Nantes, Université de Nantes, CNRS-IN2P3, Nantes, France

${ }^{35}$ Institut für Kernphysik, Johann Wolfgang Goethe-Universität Frankfurt, Frankfurt, Germany

${ }^{36}$ Laboratoire de Physique Subatomique et de Cosmologie (LPSC), Université Joseph Fourier, CNRS-IN2P3, Institut Polytechnique de Grenoble, Grenoble, France 
${ }^{37}$ Departamento de Física de Partículas and IGFAE, Universidad de Santiago de Compostela, Santiago de Compostela, Spain

${ }^{38}$ Oak Ridge National Laboratory, Oak Ridge, Tennessee, USA

${ }^{39}$ Helsinki Institute of Physics (HIP) and University of Jyväskylä, Jyväskylä, Finland

${ }^{40}$ Physics Department, University of Cape Town and iThemba LABS, National Research Foundation, Somerset West, South Africa

${ }^{41}$ Sezione INFN, Catania, Italy

${ }^{42}$ Laboratoire de Physique Corpusculaire (LPC), Clermont Université, Université Blaise Pascal,

CNRS-IN2P3, Clermont-Ferrand, France

${ }^{43}$ Gangneung-Wonju National University, Gangneung, South Korea

${ }^{44}$ Physics Department, University of Jammu, Jammu, India

${ }^{45}$ Commissariat à l'Energie Atomique, IRFU, Saclay, France

${ }^{46}$ Institute of Experimental Physics, Slovak Academy of Sciences, Kořice, Slovakia

${ }^{47}$ Institute of Physics, Bhubaneswar, India

${ }^{48}$ Dipartimento di Fisica e Astronomia dell'Università and Sezione INFN, Catania, Italy

${ }^{49}$ The Henryk Niewodniczanski Institute of Nuclear Physics, Polish Academy of Sciences, Cracow, Poland

${ }^{50}$ Joint Institute for Nuclear Research (JINR), Dubna, Russia

${ }^{51}$ Department of Physics, University of Oslo, Oslo, Norway

${ }^{52}$ Niels Bohr Institute, University of Copenhagen, Copenhagen, Denmark

${ }^{53}$ Indian Institute of Technology Bombay (IIT), Mumbai, India

${ }^{54}$ Institut Pluridisciplinaire Hubert Curien (IPHC), Université de Strasbourg, CNRS-IN2P3, Strasbourg, France

${ }^{55}$ University of Houston, Houston, Texas, USA

${ }^{56}$ Instituto de Física, Universidad Nacional Autónoma de México, Mexico City, Mexico

${ }^{57}$ Petersburg Nuclear Physics Institute, Gatchina, Russia

${ }^{58}$ Nikhef, National Institute for Subatomic Physics and Institute for Subatomic Physics of Utrecht University, Utrecht, Netherlands

${ }^{59}$ University of Tsukuba, Tsukuba, Japan

${ }^{60}$ Laboratori Nazionali di Frascati, INFN, Frascati, Italy

${ }^{61}$ Centro de Investigaciones Energéticas Medioambientales y Tecnológicas (CIEMAT), Madrid, Spain

${ }^{62}$ Kirchhoff-Institut für Physik, Ruprecht-Karls-Universität Heidelberg, Heidelberg, Germany

${ }^{63}$ Lawrence Berkeley National Laboratory, Berkeley, California, USA

${ }^{64}$ Institut für Informatik, Johann Wolfgang Goethe-Universität Frankfurt, Frankfurt, Germany

${ }^{65}$ Moscow Engineering Physics Institute, Moscow, Russia

${ }^{66}$ Institute for High Energy Physics, Protvino, Russia

${ }^{67}$ Faculty of Science, P.J. Šafárik University, Košice, Slovakia

${ }^{68}$ Wayne State University, Detroit, Michigan, USA

${ }^{69}$ Nikhef, National Institute for Subatomic Physics, Amsterdam, Netherlands

${ }^{70}$ Purdue University, West Lafayette, Indiana, USA

${ }^{71}$ Faculty of Mathematics, Physics and Informatics, Comenius University, Bratislava, Slovakia

${ }^{72}$ Russian Federal Nuclear Center (VNIIEF), Sarov, Russia

${ }^{73}$ Dipartimento di Fisica e Astronomia dell'Università and Sezione INFN, Padova, Italy

${ }^{74}$ Central China Normal University, Wuhan, China

${ }^{75}$ Sección Física, Departamento de Ciencias, Pontificia Universidad Católica del Perú, Lima, Peru

${ }^{76}$ Dipartimento di Fisica dell'Università and Sezione INFN, Trieste, Italy

${ }^{77}$ Centro de Investigación y de Estudios Avanzados (CINVESTAV), Mexico City and Mérida, Mexico

${ }^{78}$ Universidade de São Paulo (USP), São Paulo, Brazil

${ }^{79}$ Dipartimento di Fisica dell'Università and Sezione INFN, Cagliari, Italy

${ }^{80}$ Centro de Aplicaciones Tecnológicas y Desarrollo Nuclear (CEADEN), Havana, Cuba

${ }^{81}$ Yonsei University, Seoul, South Korea

${ }^{82}$ Saha Institute of Nuclear Physics, Kolkata, India

${ }^{83}$ Physics Department, Creighton University, Omaha, Nebraska, USA

${ }^{84}$ Université de Lyon, Université Lyon 1, CNRS/IN2P3, IPN-Lyon, Villeurbanne, France

${ }^{85}$ Division of Experimental High Energy Physics, University of Lund, Lund, Sweden

${ }^{86}$ Pusan National University, Pusan, South Korea

${ }^{87}$ Sezione INFN, Cagliari, Italy

${ }^{88}$ Institut de Physique Nucléaire d'Orsay (IPNO), Université Paris-Sud, CNRS-IN2P3, Orsay, France

${ }^{89}$ Dipartimento di Scienze e Innovazione Tecnologica dell'Università del Piemonte Orientale and Gruppo Collegato INFN, Alessandria, Italy

${ }^{90}$ Benemérita Universidad Autónoma de Puebla, Puebla, Mexico

${ }^{91}$ Instituto de Ciencias Nucleares, Universidad Nacional Autónoma de México, Mexico City, Mexico

${ }^{92}$ Warsaw University of Technology, Warsaw, Poland

${ }^{93}$ Institute of Space Sciences (ISS), Bucharest, Romania

${ }^{94}$ Bose Institute, Department of Physics and Centre for Astroparticle Physics and Space Science (CAPSS), Kolkata, India

${ }^{95}$ Universidade Estadual de Campinas (UNICAMP), Campinas, Brazil 
${ }^{96}$ Dipartimento di Fisica E.R. Caianiello" dell'Università and Gruppo Collegato INFN, Salerno, Italy

${ }^{97}$ Sezione INFN, Bari, Italy

${ }^{98}$ National Centre for Nuclear Studies, Warsaw, Poland

${ }^{99}$ Sezione INFN, Rome, Italy

${ }^{100}$ Chicago State University, Chicago, Illinois, USA

${ }^{101}$ Institute for Nuclear Research, Academy of Sciences, Moscow, Russia

${ }^{102}$ Physics Department, University of Athens, Athens, Greece

${ }^{103}$ Sezione INFN, Trieste, Italy

${ }^{104}$ Universidad Autónoma de Sinaloa, Culiacán, Mexico

${ }^{105}$ Physics Department, University of Rajasthan, Jaipur, India

${ }^{106}$ Technical University of Split FESB, Split, Croatia

${ }^{107}$ A. I. Alikhanyan National Science Laboratory (Yerevan Physics Institute) Foundation, Yerevan, Armenia ${ }^{108}$ University of Tokyo, Tokyo, Japan

${ }^{109}$ Department of Physics, Sejong University, Seoul, South Korea

${ }^{110}$ Eberhard Karls Universität Tübingen, Tübingen, Germany

${ }^{111}$ KTO Karatay University, Konya, Turkey

${ }^{112}$ Zentrum für Technologietransfer und Telekommunikation (ZTT), Fachhochschule Worms, Worms, Germany

${ }^{113}$ Technische Universität München, Munich, Germany

${ }^{114}$ California Polytechnic State University, San Luis Obispo, California, USA

${ }^{115}$ The University of Texas at Austin, Physics Department, Austin, Texas, USA

${ }^{116}$ Vestfold University College, Tonsberg, Norway

${ }^{117}$ Nuclear Physics Group, STFC Daresbury Laboratory, Daresbury, United Kingdom

${ }^{118}$ Institut für Kernphysik, Technische Universität Darmstadt, Darmstadt, Germany

${ }^{119}$ M.V.Lomonosov Moscow State University, D.V.Skobeltsyn Institute of Nuclear Physics, Moscow, Russia

${ }^{120}$ Institute of Physics, Academy of Sciences of the Czech Republic, Prague, Czech Republic

${ }^{121}$ University of Tennessee, Knoxville, Tennessee, USA

${ }^{122}$ Indian Institute of Technology Indore, Indore, India (IITI)

${ }^{123}$ Dipartimento di Fisica dell'Università La Sapienza" and Sezione INFN, Rome, Italy

${ }^{124}$ University of Belgrade, Faculty of Physics and Vinča” Institute of Nuclear Sciences, Belgrade, Serbia

${ }^{125}$ National Institute of Science Education and Research, Bhubaneswar, India

${ }^{126}$ Academy of Scientific Research and Technology (ASRT), Cairo, Egypt

${ }^{127}$ Budker Institute for Nuclear Physics, Novosibirsk, Russia

${ }^{128}$ Institute of Theoretical Physics, University of Wroclaw, Wroclaw, Poland

${ }^{129}$ Laboratori Nazionali di Legnaro, INFN, Legnaro, Italy

${ }^{130}$ Hiroshima University, Hiroshima, Japan

${ }^{131}$ Centre de Calcul de l'IN2P3, Villeurbanne, France

*Deceased. 